\title{
Research On Computer Intrusion Detection Based On Biotechnology
}

\author{
Zun DAI
}

\author{
College of agriculture Hainan University
}

\begin{abstract}
Keywords: Computer technology, intrusion detection, gene engineering, biotechnology
\end{abstract}
\begin{abstract}
With the continuous improvement of computer performance, the volume decreases, the continuous use of simplified network more and more convenient use, now computer technology rapid detection of intrusion has penetrated into almost all areas. Some people say that twenty-first Century is the era of biotechnology, since entering twenty-first Century the world biotechnology has achieved great development, but because the computer is becoming more and more advanced, which makes the computer can do more for biotechnology can not complete the work. However, the development of biotechnology also provides a powerful driving force for the rapid development of computer intrusion detection technology. Therefore, the rapid detection of biological technology and computer technology has now reached a point of mutual complement. This paper mainly introduces the technology of the computer intrusion and rapid detection of application in molecular biotechnology, biotechnology, medical technology and other biological technology, promote the development of the computer technology for rapid detection of intrusion and biological technology.
\end{abstract}

\section{Introduction}

With the continuous improvement of computer performance, the volume decreases, the continuous use of simplified network more and more convenient use, now computer technology rapid detection of intrusion has penetrated into almost all areas. Some people say that twenty-first Century is the era of biotechnology, since entering twenty-first Century the world biotechnology has achieved great development, but because the computer is becoming more and more advanced, which makes the computer can do more for biotechnology can not complete the work. However, the development of biotechnology also provides a powerful driving force for the rapid development of computer intrusion detection technology. Therefore, the rapid detection of biological technology and computer technology has now reached a point of mutual complement. The computer is widely used in the fields of scientific computing, process detection and control, information management, computer aided system and so on.

\section{The application of computer technology in the research of molecular biology}

With the development of molecular biotechnology, the application of computer technology in the research of gene mapping and sequencing is becoming more and more important. Now, the world's molecular biologists are working on the largest ever data collection effort. In the country, schools, research institutes and enterprises belong to the laboratory technology researchers are from the lowest to the determination of the entire genome sequence of bacteria and the higher people's determination to find the mapping work, has the economic value of genetic information in the new development and the use way. By the end of the century, molecular biologists hope to get the genome of tens of thousands of biotechnology. It will be a huge database of evolutionary "blueprints" for many plants, animals, and microbes that are distributed in different parts of the earth. However, the amount of information generated by it is beyond our imagination, of course, we will not be able to use a pen, paper to manage and access. For such a large amount of information generated by biological technology, we can only manage through the research on the rapid detection technology of computer intrusion, electronically stored in distributed in various countries and regions of the world in the database. Collection, download, management and use of genomic information will require rapid 
detection of intrusion between computer technology and biotechnology to cooperate more closely, but also requires researchers in physics, mathematics, engineering, computer science, chemistry and molecular biotechnology and other fields to carry out comprehensive training.

The human genome project has accelerated the integration of computer intrusion detection and genetic engineering. Without the help of computer scientists and the increasingly sophisticated computer technology to detect intrusions, it would be impossible to determine and analyze the sequence of base pairs in human cells. The human genome project is transforming biotechnology into information science. Many biological scientists feel that the process of obtaining a sequence is boring, but from a computer science perspective, this is the first class of challenging algorithmic problems. Genome mapping and sequencing work has just begun. The natural world re arranged at the genetic level, and focus on transforming it into useful network products on the market, is a daunting challenge, this is undoubtedly a huge human once unimaginable management tasks. Understanding and description of all the genes between, between organizations, organs, between species and environment relationship network, and causes changes in the genetic mutation and phenotype, any complex system has studied the cannot handle. It is only through an interdisciplinary approach, in particular, that information scientists rely on computational skills to achieve this task.

\section{The application of computer technology in the field of Biotechnology}

Biotechnology is the application of modern science and technology, the principles and methods of science, from the engineering point of view of the human body structure, function and its relationship and other life phenomena. Its purpose is to solve the biomedical problems, namely the research and development of disease prevention and treatment, as well as the function of human body, such as biomedical devices and systems. The concept and method of scientific technology are used to explain and describe the components, structures and functions of the human body.

(1), The application of computer aided in biomedicine: computer assisted therapy in clinical application is mainly embodied in computer assisted surgery (computer-aided surgery CAS) technology, CAS technology through space biomedical imaging and advanced positioning technology combined with rapid detection of the invasion of computer technology especially graphic image processing technology, auxiliary the doctor made reasonable operation plan, accurate guide operation safety. With the continuous pursuit of modern surgery for the individualization of treatment, the accuracy of treatment and the limited trauma, CAS technology has been widely used in clinic, especially in Department of Neurosurgery, Department of orthopedics and Department of ENT.

Computer aided design (CAD-Computer Aided Design) is the use of computers and graphics equipment to help designers to design work, referred to as cad. In the design of different schemes are usually a large number of computer calculation, analysis and comparison, to determine the optimal solution; a variety of design information, whether digital, text or graphics, can be stored in a computer memory or external memory, and can quickly retrieve; designers usually start with a sketch design the sketch into a heavy work, working drawing can be done to the computer; the design results automatically generated by the computer, can make fast graphics display, allowing designers to make timely judgments and modify the design; the computer can be carried out with graphics editing, zoom, rotation and translation of graphics data processing work. CAD can reduce the repetitive work of designers, such as drawing, focusing on the design itself, shorten the design cycle and improve design quality. At present, computer aided design has been widely used in prosthodontics, prosthetic design and so on.

(2) The application of computer information management in hospitals: hospital management system is the use of computer and communications equipment for all hospital departments to provide patient care information and administration information collection, storage, processing, extraction and data exchange capabilities, and meet the needs of all authorized users. The system realizes the dynamic monitoring of hospital patient doctor and medicine circulation of the whole process of management with the computer, the traditional rely on manual operation, heavy workload, low efficiency, easy to large amounts of data error can be used instead of computer processing, greatly 
reducing the management workload, but also can provide a complete data from patients, provide convenient and fast for patients.

\section{The promotion of the development of biotechnology on the rapid detection of computer intrusion detection technology}

At present, with the rapid development of computer industry, the research of computer technology intrusion detection is becoming more and more mature. However, the density of traditional computer transistors we use is close to the theoretical limit of the current technology. Therefore, in order to achieve greater development in the computer, people need to constantly look for new computer architecture.

Scientists in order to achieve high degree of integration, make the computer get further development, they turned to biotechnology is on the rise, and the reference to all kinds of processing problem of the biotechnology industry way, put forward some new biological technology computer model. In the past more than half a century, molecular biotechnology has broken down the phenomenon of life into a large number of genes and proteins. International famous magazine "Nature" has been reported that the University of Cambridge, the study found that the biological circuit". They studied a protein found in bacteria, bacteria exist in the information processing network composed of proteins, the network may change according to the molecular density and shape properties of the transmission and processing of information, and to drive the bacteria swim to where nutrients place according to the received information. Similarly, experts at the Stanford University in bacteria were also found in the "biotechnology circuit", and logic phenomenon was found in the energy of glycolysis by biological technology solution process, find the relevant logic gate "'. Therefore, human beings can use genetic engineering technology, imitation of the protein molecules, used as components of the computer. Since the 70s of last century, it has been found that DNA (deoxyribonucleic acid) in different states, can produce information and no information changes. Associated with the logic circuit of 0 and 1, transistor conduction or cut-off, high or low voltage, pulse signal with or without, and so on, scientists inspired the development of bio inspired technology components. On this basis, in 1995, from around the world more than 200 experts to discuss the feasibility of DNA computer, that DNA molecules under the action of the enzyme by bio chemical reaction technology can be transformed from a gene code for another gene code transformation gene code can be used as input data, the genetic code after the reaction the calculation results can be used as. Using this process can also be made into a new type of biotechnology computer. At present, the latest generation of experimental biotechnology computer is simulating the human brain. People are trying to find the similarity between the neuron and the silicon chip.

\section{References}

[1] Liang Yubing; colorimetric method for rapid detection of coliform bacteria in food [D]; Jilin University; 2012

[2] Li Xiaojie; automatic focusing technology of automatic detection system for food microbiology [D]; Jilin University;

[3] the history of Hongwei microscopic image processing; [D] theory and its application in Food Microbiology Identification; Jilin University; 2012

[4] Shi Hongwei; Shi Wu Wu; Yin Yongguang; automatic detection method of food bacteria based on image processing [J]; Journal of Jilin University (Engineering Science Edition); 2012 issue of 04

[5] Ding Jun; Yin Yongguang; points; the number of coliform bacteria [J] spectrophotometric method for rapid detection of food; food technology; 201214 